\title{
Automated 2D Segmentation of Prostate in T2-weighted MRI Scans
}

\author{
J. Jucevičius, P. Treigys, J. Bernatavičienè, R. Briedienè, \\ I. Naruševičiūtè, G. Dzemyda, V. Medvedev
}

\begin{abstract}
Justinas Jucevičius*, Povilas Treigys, Jolita Bernatavičienė, Gintautas Dzemyda, Viktor Medvedev

Vilnius University, Institute of Mathematics and Informatics

Lithuania, 08663 Vilnius, Akademijos str. 4

justinas.jucevicius@mii.vu.lt, povilas.treigys@mii.vu.lt, jolita.bernataviciene@mii.vu.lt, gintautas.dzemyda@mii.vu.lt, viktor.medvedev@mii.vu.lt

*Corresponding author: justinas.jucevicius@mii.vu.lt
\end{abstract}

\section{Rūta Briedienè, Ieva Naruševičiūtè}

Vilnius University, National Cancer Institute

Lithuania, 08660 Vilnius, Santariškiụ str. 1

ruta.briediene@nvi.lt, ieva.naruseviciute@gmail.com

\begin{abstract}
The prostate cancer is the second most frequent tumor amongst men. Statistics shows that biopsy reveals only $70-80 \%$ clinical cancer cases. Multiparametric magnetic resonance imaging (MRI) technique comes to play and is used to help to determine the location to perform a biopsy. With the aim to automating the biopsy localization, prostate segmentation has to be performed in magnetic resonance images. Computer image analysis methods play the key role here. The problem of automated prostate magnetic resonance (MR) image segmentation is burdened by the fact that MRI signal intensity is not standardized: field of view and image appearance is for a large part determined by acquisition protocol, field strength, coil profile and scanner type. Authors overview the most recent Prostate MR image segmentation challenge results and provide insights on T2-weighted MRI scan images automated prostate segmentation problem by comparing the best obtained automatic segmentation algorithms and applying them to $2 \mathrm{D}$ prostate segmentation case. The most important benefit of this research will have medical doctors involved in the management of the cancer.
\end{abstract}

Keywords: computer image processing, 2D prostate segmentation, magnetic resonance imaging (MRI), T2-weighted scan.

\section{Introduction}

Various data mining methods find application in medicine and health care. Large studies are presented in [9], [22], there are a lot of recent applications, eg. [7], [8], [25], [27]. This paper deals with medical image analysis, particularly with magnetic resonance images and prostate cancer. World Cancer Research Fund International states that prostate cancer is the second most frequent tumor among men and fourth most common among both genders. Lithuanian cancer registry data from 2012 shows that prostate cancer prevalence reaches $34 \%$ amongst men aging 55 to 74 years. The mortality from the prostate cancer is the second most common after the lung cancer amongst men and the third most common among both genders after lung and stomach cancers [23]. According to European Association of Urology guidelines, from 10 to 12 core biopsy is recommended in case of prostate-specific antigen level elevation and/or suspicious digital rectal examination findings [1]. Contemporary, random systematic prostate biopsy strategy includes failure to detect clinically significant cancer. Undersampling in up to $30 \%$ of cases 
with clinically significant tumors being missed on initial biopsy. This diagnostic uncertainty can lead to repeat biopsy, delayed detection of significant disease and disease overtreatment [20]. Despite inaccuracy, biopsy remains the main way that can unambiguously detect prostate cancer if performed on the right location.

The latest recommendations in prostate cancer care include multiparametric magnetic resonance imaging (mpMRI) as the tool for prostate cancer diagnosis, characterization, staging as well as risk stratification among men, who need active surveillance. Today's computer-aided detection programs are associated with European Society of Urogenital recommendations for prostate evaluation by mpMRI are the attractive subject for research that incorporates the development of new image analysis [4], [16], [26] and data mining algorithms. Usually prostate localization and segmentation in magnetic resonance images is done by hand; however, it takes a lot of time and can be inaccurate. This causes the need for software to aid in automated prostate segmentation [13] in a standardized manner. Thus the main objective of this study was to overview the current situation of the field and to adopt today's best methods developed to a procedure named Prostate Template Biopsy [30].

\section{Research Motivation and Experiment Setup}

The problem of automated prostate MR image segmentation is burdened by the fact that most researchers cannot compare the effectiveness of different algorithms due to either troublesome implementation without the help of the original author or algorithm being closed source. What further aggravates the problem is that MRI signal intensity is not standardized and image appearance is for a large part determined by acquisition protocol, field strength, coil profile, and scanner type [18].

It is challenging task to identify and segment objects within images due to high object and background variability. In computer vision image segmentation can be described as procedure of finding group of image pixels that shares the same feature and describe homogeneous image region. Analysis may take into account object texture, intensities shape and etc [3]. However, the problem arises when investigative object is compound of several regions, object edges are blurred or object's shape is varying. Here the segmentation techniques are applied that makes further processing easier: to each group of pixels that describes the region the unique region label is assigned.

Researchers [10] have put a big effort to summarize prostate segmentation methods. Study reveals four different groups of segmentation algorithms that fall into:

- Contour and shape based methods exploit contour and shape information to accomplish segmentation task.

- Region-based methods analyses predominant prostate intensity distribution in different modalities.

- Supervised and unsupervised classification methods aim at obtaining a partition of the feature space into a set of labels for different regions. For this task classifier and/or clustering techniques are used.

- Hybrid methods combine a priori boundary, shape, region, and feature information of the prostate gland. Methods of this group are robust to noise and produce superior results in presence of shape and texture variations.

Several successful Grand Challenges in Medical Imaging have been organized in recent years to deal with similar issues in the fields of coronary image analysis, retinal image analysis, liver 
segmentation on computed tomography (CT) scan, lung registration on CT scan, brain segmentation on MRI and prostate segmentation on MRI. Prostate MR Image Segmentation challenge (PROMISE12) [18] was designed to allow comparison of segmentation algorithms on the basis of robustness and performance by providing hundred T2-weighted MRI scans gathered from four different institutions. T2-weighted MR images were used because they contain most anatomical detail and most current researchers focus on them for segmentation. With the aim to evaluate segmentation results the PROMISE12 challenge introduce those widely used metrics:

- dice similarity coefficient (DSC) [15],

- absolute relative volume difference [12],

- average boundary distance [12],

- $95 \%$ Hausdorff distance (HD) [5].

The score for each metric was mapped to a relative value between 0 and 100 . The scores for all metrics were then averaged to obtain a score per case, and the average of score over all cases was calculated and used to rank algorithms. Table 1 presents prostate detection algorithms and depicts obtained results of the challenge. Here, A stands for an automatic method that requires no user interaction; S - semi-automatic method that requires some initial user interaction; I interactive method that requires full user interaction from the beginning of segmentation until the end.

Table 1: Prostate detection algorithms [2]

\begin{tabular}{l|l|l|l}
\hline Team name & Score & Type & The idea of the algorithm \\
\hline Imorphics & 84.36 & $\mathrm{~A}$ & $\begin{array}{l}\text { Apply active appearance models on images with increasing reso- } \\
\text { lution by refining results }\end{array}$ \\
\hline ScrAutoProstate & 83.49 & $\mathrm{~A}$ & $\begin{array}{l}\text { Intensity normalization, marginal space learning, boundary refine- } \\
\text { ment }\end{array}$ \\
\hline CBA & 80.66 & $\mathrm{I}$ & $\begin{array}{l}\text { Smart paint algorithm segments prostate by sweeping the mouse } \\
\text { cursor in the object or background }\end{array}$ \\
\hline SBIA & 78.34 & $\mathrm{~A}$ & Multi-atlas based segmentation, zooming into vicinity \\
\hline Grisles & 77.56 & $\mathrm{~S}$ & $\begin{array}{l}\text { Detect location of the prostate, use probabilistic active shape } \\
\text { model for boundary detection }\end{array}$ \\
\hline Robarts & 77.32 & $\mathrm{~S}$ & $\begin{array}{l}\text { Contour evolution with the integration of the generic star shapes } \\
\text { prior }\end{array}$ \\
\hline ICProstateSeg & 76.06 & $\mathrm{~A}$ & $\begin{array}{l}\text { Multi-atlas based segmentation using local appearance-specific at- } \\
\text { lases }\end{array}$ \\
\hline Utwente & 75.23 & $\mathrm{~S}$ & Active appearance models based segmentation \\
\hline Cimalab & 74.68 & $\mathrm{~A}$ & $\begin{array}{l}\text { Atlas-based segmentation that selects the most similar templates } \\
\text { using multi-scale SURF analysis and applies linear combination }\end{array}$ \\
\hline DIAG & 73.30 & $\mathrm{~A}$ & $\begin{array}{l}\text { Multi-atlas based segmentation using selective and iterative } \\
\text { method for performance level estimation algorithm for merging } \\
\text { atlas labels }\end{array}$ \\
\hline ETHZ & 72.38 & $\mathrm{~A}$ & $\begin{array}{l}\text { Graph cut based segmentation using image features, context in- } \\
\text { formation, semantic knowledge }\end{array}$ \\
\hline Rutgers & 70.44 & $\mathrm{~S}$ & $\begin{array}{l}\text { Random decision-based forest for classification and the propaga- } \\
\text { tion of region for segmentation }\end{array}$ \\
Segmentation based on active appearance models
\end{tabular}

Two best fully automated algorithms from the PROMISE12 challenge named Imorphics [28], [11] and ScrAutoProstate [2] having scores of 84.36 and 83.49 respectively were chosen for further analysis. The pipelines of the Imorphic and ScrAutoProstate methods are presented in Figure 1. 

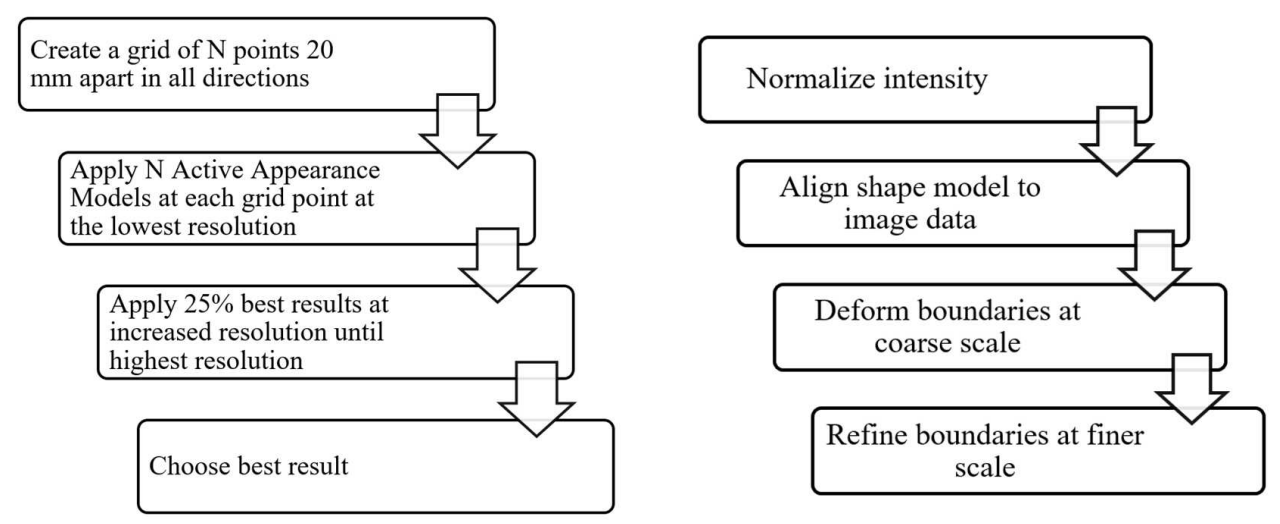

Figure 1: Pipelines of the Imorphics (on the left side) and ScrAutoProstate (on the right side) prostate segmentation methods

Both algorithms were adapted to be suitable for prostate segmentation in 2D space instead of the 3D space originally used in the challenge. All adaptations were performed by choosing the corresponding algorithm designed for 2D space without any modifications. Prostate segmentation in 2D space was chosen because of the biopsy procedure named Prostate Template Biopsy [30]:

- It has an accuracy of $95 \%$.

- It is becoming more and more prevalent.

- It uses only two slices out of 10-40 used in 3D space, thus reduces complexity and computational time needed for segmentation.

The Imorphics method [11], [28] belongs to a group of hybrid prostate segmentation methods. The method converts voxel-based segmentation to surface by using the marching-cube algorithm [19]. Segmentation allows indicating whether surface belongs to the prostate gland or not. Next, to construct statistical prostate appearance model, so called mean image, a set of possible deformations are introduced and registered together [6]. This step allows finding the minimum information needed to code the mean reference image with the deformations that map mean image to each example image. Finally, to obtain features of the image authors use active appearance model that control shape and texture. The model computes the closest match of the prostate gland shape in sample image using the least squares sum of residuals. For the efficiency Jacobian matrix describing the average change in residuals with respect to changes in model parameters on a training set is pre-computed and for the initial estimate of the model, authors introduce grid of starting search points across the image, typically $20 \mathrm{~mm}$ apart in all directions.

At the initial stage the ScrAutoProstate method [2] applies region based brightness and contrast Poison editing technique [21]. Then, with the view to constructing statistical shape model, training segmentation mask (represented as a mesh) is constructed the same way as it was presented in the Imorphics method by using the marching cube technique. Then, orientation and scale variations in those statistical shapes are removed after application of Procrustes analysis [14]. The remaining shape variability is finally represented with a point distribution model, and the strongest shape models are extracted through principal component analysis. While testing algorithm with the unseen image, initial segmentation is obtained by applying teh Marginal Space Learning [29] algorithm that computes unknown pose and shape coefficients. The previous step aligns shape model to the image and gives good initial segmentation. At the final step, mesh surface is refined by using non-rigid, hierarchical boundary deformation [17]. Introduced refinement iteratively displaces mesh vertices along the mesh surface normal. 


\section{Experiments and Results}

In this research, we have used images from 50 cases provided by the PROMISE12 [18] challenge. Initial three-dimensional MRI data set used by the challenge was split into separate images representing every slice. The number of slices per case varied from 15 to 54 . Images that did not contain prostate were removed. Remaining images were split into two groups representing prostate apex and base parts. From each group, middle image was chosen to represent the apex and base part of the prostate respectively having 100 images in total. All images were gathered from four different institutions and varied in resolution:

- 256 x 256 pixels;

- 320 x 320 pixels;

- 512 x 512 pixels.

To test the performance of the 2D prostate segmentation algorithms we have used leave-oneout cross-validation [24], where each image is segmented using a model, built from the training set with this image removed, i.e. image is "excluded" from the set of images and used for validation. The result was then compared against the reference segmentation. Results, presented in Table 2 , show that transition from 3D to 2D space prostate gland segmentation can be accomplished with the minimum loss of accuracy. DSC measure when compared Imorphic and ScrAutoProstate algorithms decreased by 0.04 and 0.01 points respectively. It results that $2 \mathrm{D}$ segmentation is successful and opens the possibility to apply Prostate Template Biopsy procedure that unifies biopsy of the prostate gland procedure.
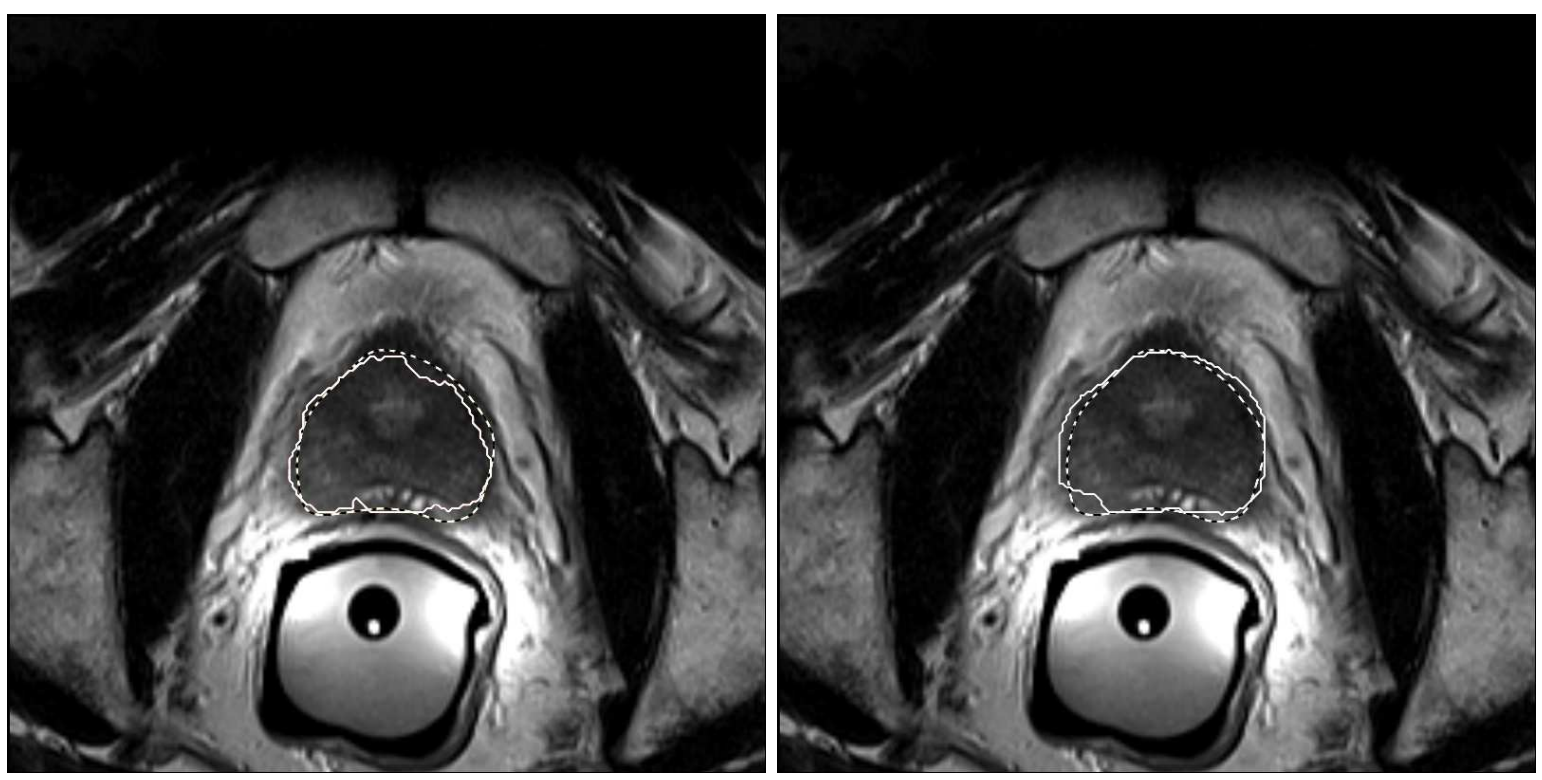

Figure 2: Segmentation results in 2D space of both Imorphics and ScrAutoProstate methods on the left and right respectively. In dashed line - reference segmentation, in white line - automated segmentation.

DSC and 95\% Hausdorff distance statistics were selected to match those reported in original algorithms for comparison. The DSC measures the amount of overlap between the reference segmentation and the automated segmentation. DSC can range from zero to one, where zero represents no overlap and one corresponds to identical segmentations. The directed HD identifies 
Table 2: Comparison of prostate segmentation results between 2D and 3D space by analyzing $\mathrm{T} 2$-weighted scan data

\begin{tabular}{l|l|l|l|l}
\hline \multirow{2}{*}{ Algorithm/Measures } & \multicolumn{2}{|c|}{ Imorphics } & \multicolumn{2}{c}{ ScrAutoProstate } \\
\cline { 2 - 5 } & 2D & 3D & 2D & 3D \\
\hline DSC & 0.84 & 0.88 & 0.83 & 0.82 \\
\hline HD & 6.26 & 4.17 & 9.73 & not specified \\
\hline Execution Time (s) & 51.11 & 480 & 0.24 & 2.30 \\
\hline
\end{tabular}

the point on the reference segmentation that is the farthest from any point on the model segmentation and measures the distance from this point to the nearest point on the model segmentation. The $95 \%$ HD is less sensitive to outliers than HD since it considers the point representing the $95^{\text {th }}$ percentile of the distances instead of the farthest. Figure 2 shows sample segmentation results. Visually, both algorithms produces quite similar segmentation results. Metrics measures are as follows: Imorphics DSC and HD values were 0.93 and 4.12; ScrAutoProstate DSC and HD values were 0.93 and 6.99 .

\section{Conclusions}

3D prostate gland segmentation cannot be directly adapted to today's best methods developed to a procedure named Prostate Template Biopsy. That leads to an investigation whether $3 \mathrm{D}$ prostate gland segmentation can be transferred to $2 \mathrm{D}$ segmentation while keeping the same segmentation accuracy with the possibility to speed up algorithms execution time. 2D images representing slices are starting point for the analysis and further conclusions.

The investigation presented in this paper has shown that there is a minor loss in algorithms accuracy when moving prostate gland segmentation from 3D space to $2 \mathrm{D}$ space. Dice similarity coefficient when compared Imorphics and ScrAutoProstate algorithms have changed by 0.04 and 0.01 points, respectively. However, Imorphics performed slightly better at the cost of execution time.

As expected, both algorithms improved execution time by almost 10 times in 2D in comparison to 3D. Despite both algorithms perform quite well, the development is necessary for practical usage where automated prostate segmentation in MRI is needed. The most important benefit of this research will have medical doctors involved in the management of the cancer: radiologists, urologists, histopathologists, radiotherapists, oncologists.

\section{Bibliography}

[1] Archip, N., Clatz, O., Whalen, S., Kacher, D., Fedorov, A., et al. (2007), Non-rigid alignment of preoperative MRI, fMRI, and DT-MRI with intra-operative MRI for enhanced visualization and navigation in image-guided neurosurgery, NeuroImage, DOI: 10.1016/j.neuroimage.2006.11.060

[2] Birkbeck, N., Zhang, J., Requardt, M., Kiefer, B., Gall, P., Kevin Zhou, S., (2012), Regionspecific hierarchical segmentation of MR prostate using discriminative learning, Proc. Med. Image Comput. Comput.-Assisted Intervention Conf. Prostate Segment. Challenge, 4-11.

[3] Borenstein, E., Malik, J. (2006, June). Shape guided object segmentation. In 2006 IEEE Computer Society Conference on Computer Vision and Pattern Recognition (CVPR'06), 1: 969-976. 
[4] Buteikienè, D., Paunksnis, A., Barzdžiukas, V., Bernatavičienè, J., Marcinkevičius, V., Treigys, P. (2012); Assessment of the optic nerve disc and excavation parameters of interactive and automated parameterization methods. Informatica, 23(3): 335-355.

[5] Chandra, S., Dowling, J., Shen, K., Raniga, P., Pluim, J., Greer, P., Salvado, O., Fripp, J. (2012); Patient specific prostate segmentation in 3-D magnetic resonance images. IEEE Trans. Med. Imaging, 31: 1955-1964.

[6] Cootes, T., Petrovi, C., Schestowitz, R., Taylor, C. (2005); Groupwise construction of appearance models using piece-wise affine deformations. 16th British Machine Vision Conference, 2:879-888.

[7] Costin, H., Bejinariu, S., \& Costin, D. (2016); Biomedical Image Registration by means of Bacterial Foraging Paradigm. International Journal of Computers Communications 8$\}$ Control, 11(3): 331-347.

[8] Dua, S., \& Acharya, R. (Eds.). (2016); Data Mining in Biomedical Imaging, Signaling, and Systems, CRC Press, 2016.

[9] Ghavami, P.K. (2014). Clinical Intelligence the Big Data Analytics Revolution in Healthcare: A Framework for Clinical and Business Intelligence Createspace Independent Pub., 2014.

[10] Ghose, S., Oliver, A., Marti, R., Llado, X., Vilanova, J., et al. (2012); A Survey of Prostate Segmentation Methodologies in Ultrasound, Magnetic Resonance and Computed Tomography Images, Computer Methods and Programs in Biomedicine, Elsevier, hal-00695557, 2012.

[11] Graham, V., Gwenael, G., Mike, B. (2012); Fully Automatic Segmentation of the Prostate using Active Appearance Models, PROMISE12 challenge website.

[12] Heimann, T. et al. (2009); Comparison and Evaluation of Methods for Liver Segmentation From CT Datasets, IEEE Transactions on Medical Imaging, 28(8): 1251-1265, DOI: 10.1109/TMI.2009.2013851.

[13] Hemanth, D. J., Anitha, J., \& Balas, V. E. (2015); Performance Improved Modified Fuzzy C-Means Algorithm for Image Segmentation Applications. Informatica, 26(4): 635-648.

[14] Kendall, D. (1989); A Survey of the Statistical Theory of Shape. Statistical Science, 4(2): 87-99.

[15] Klein, S., van der Heide,I., Lips, M., van Vulpen, M., Staring, M., Pluim, J. (2008); Automatic segmentation of the prostate in 3D MR images by atlas matching using localized mutual information; Med. Phys, 35(4):1407-1417.

[16] Lekas, R.et al. (2008); Monitoring changes in heart tissue temperature and evaluation of graft function after coronary artery bypass grafting surgery. Medicina, 45(3): 221-225.

[17] Ling, H., Zhou, S.K., Zheng, Y., Georgescu, B., Suehling, M., Comaniciu, D. (2008); Hierarchical, learning-based automatic liver segmentation. CVPR, IEEE Computer Society, 1-8.

[18] Litjens, G., Toth, R., van de Ven, W., Hoeks, C., Kerkstra, S., et al. (2014); Evaluation of prostate segmentation algorithms for MRI: The PROMISE12 challenge, Medical Image Analysis, 18(2):359-373.

[19] Lorensen, W., Cline, H. (1987); Marching cubes: A high resolution 3d surface construction algorithm. SIGGRAPH Comput. Graph., 21(4): 163-169. 
[20] Mottet, N., Bellmunt, J., Briers, E., Bergh, R., Bolla, M., Casteren, N., e. al. (2015); Guidelines on prostate cancer. European Association of Urology, 2015.

[21] Perez, P., Gangnet, M., Blake, A. (2003); Poisson image editing, ACM Trans. Graph. 22(3): 313-318.

[22] Reddy, C. K., \& Aggarwal, C. C. (Eds.). (2015); Healthcare data analytics, CRC Press, 2015 .

[23] Smailytė, G., Aleknavičienè, B. (2015); Vëžys Lietuvoje 2012 m. Nacionalinio véžio instituto véżio kontrolès ir profilaktikos centras, 2015.

[24] Sylvain, A., Alain, C. (2010); A survey of cross-validation procedures for model selection. Statist. Surv, 4: 40-79.

[25] Termenon, M., Grana, M., Savio, A., Akusok, A., Miche, Y., Bjork, K. M., \& Lendasse, A. (2016); Brain MRI morphological patterns extraction tool based on Extreme Learning Machine and majority vote classification. Neurocomputing, 174: 344-351.

[26] Treigys, P., Šaltenis, V., Dzemyda, G., Barzdžiukas, V., \& Paunksnis, A. (2008); Automated optic nerve disc parameterization, Informatica, 19(3): 403-420.

[27] Trigui, R., Miteran, J., Sellami, L., Walker, P., \& Hamida, A. B. (2016); A classification approach to prostate cancer localization in 3T multi-parametric MRI, Advanced Technologies for Signal and Image Processing (ATSIP), 2016 2nd International Conference on, IEEE, 113-118.

[28] Vincent, G., Guillard, G., Bowes, M. (2012); Fully automatic segmentation of the prostate using active appearance models, MICCAI Grand Challenge: Prostate MR Image Segmentation, 2012.

[29] Zheng, Y., Barbu, A., Georgescu, B., Scheuering, M., Comaniciu, D. (2008). Four-chamber heart modeling and automatic segmentation for 3-D cardiac CT volumes using marginal space learning and steerable features. IEEE Trans. Med. Imag. 27(11): 1668-1681.

[30] Prostate Template Biopsy. Essexurology.co.uk. Retrieved 2016 July 30, 2016. from http://www.essexurology.co.uk/prostate_template_biopsy.php. 